\title{
Sums and Differences of Two Cubic Polynomials
}

\author{
By \\ Trevor D. Wooley* \\ University of Michigan, Ann Arbor, USA \\ (Received 18 January 1999; in revised form 17 May 1999)
}

\begin{abstract}
When $f(x)$ is a cubic polynomial with integral coefficients, we show that almost all integers represented as the sum or difference of two values of $f(x)$, with $x \in \mathbb{Z}$, are thus represented essentially uniquely.
\end{abstract}

1991 Mathematics Subject Classification: 11P05

Key words: Cubic polynomials, sums of cubes, Waring's problem

\section{Introduction}

A sieve method of Hooley [4] shows that the representation of a number as the sum of two non-negative integral cubes is almost always unique. Let $\nu(x)$ denote the number of positive integers not exceeding $x$ that have at least two essentially distinct representations as the sum of two non-negative cubes. Then the sharper estimate subsequently obtained by Hooley in [5] shows that $\nu(x)=O_{\varepsilon}\left(x^{5 / 9+\varepsilon}\right)$. More recently, Wooley [6] has described an elementary proof of the latter estimate which avoids the use of sieve methods, and this method has been refined by HEATHBROwn [3] to show that $\nu(x)=O_{\varepsilon}\left(x^{4 / 9+\varepsilon}\right)$. In common with the earlier sieve method of Hooley [5], the argument applied by HEATH-Brown [3] makes essential use of the homogeneity of the associated polynomial $x_{1}^{3}+x_{2}^{3}-x_{3}^{3}-x_{4}^{3}$, and thus neither of the latter methods is directly capable of addressing corresponding inhomogeneous questions concerning sums of two cubic polynomials. In this paper we investigate sums and differences of a given cubic polynomial $f(x)$ through the affine slicing method of Wooley [6], deriving estimates of quality similar to those of Hooley [5] and Wooley [6] in this inhomogeneous situation. In particular, we show that the representation of a number as the sum or difference of two integral values of a cubic polynomial is almost always unique.

In order to describe our conclusions we require some notation. When $f(t)=a t^{3}+b t^{2}+c t+d$ is a cubic polynomial with integral coefficients, and $x$ is a positive real number, we define $\nu_{f}^{ \pm}(x)$ to be the number of integers $n$ with $|n| \leqslant x$ that have at least two essentially distinct representations in the form $n=f\left(x_{1}\right) \pm f\left(x_{2}\right)$, for some integers $x_{1}$ and $x_{2}$. By "essentially distinct representation", we mean a representation which does not arise from the original

\footnotetext{
* Packard Fellow and supported in part by NSF grant DMS-9622773
} 
representation by means of an automorphism of the cubic polynomial $f(x) \pm f(y)$. In this context we note that the cubic polynomial $f(x)+f(y)$ has the unique nontrivial automorphism $(x, y) \rightarrow(y, x)$ over $\mathbb{Z}$, and the polynomial $f(x)-f(y)$ has no such non-trivial automorphisms unless $3 a \mid 2 b$, in which case the latter polynomial possesses the unique automorphism $(x, y) \rightarrow(-y-2 b /(3 a),-x-2 b /(3 a))$. In Sect. 3 below we establish the following estimate for $\nu_{f}^{ \pm}(x)$.

Theorem 1. One has $\nu_{f}^{ \pm}(x)=O_{\varepsilon, f}\left(x^{5 / 9+\varepsilon}\right)$.

As with previous treatments of sums of two cubes, we establish Theorem 1 by estimating the number of non-trivial solutions of a suitable auxiliary equation. Two further consequences of the estimate developed in Sect. 2 to establish Theorem 1 are worthy of mention. When $f(t)$ is the cubic polynomial described above, and $P$ is a positive real number, denote by $S_{f}(P)$ the number of integral solutions of the equation

$$
f\left(x_{1}\right)+f\left(x_{2}\right)=f\left(x_{3}\right)+f\left(x_{4}\right),
$$

with $\left|x_{i}\right| \leqslant P(1 \leqslant i \leqslant 4)$. Also, let $T_{f}(P)$ denote the number of integral 4-tuples $\mathbf{x}$ satisfying one of the conditions

$$
\left\{x_{1}, x_{2}\right\}=\left\{x_{3}, x_{4}\right\} \quad \text { and } 3 a\left(x_{1}+x_{2}\right)+2 b=3 a\left(x_{3}+x_{4}\right)+2 b=0,
$$

with $\left|x_{i}\right| \leqslant P(1 \leqslant i \leqslant 4)$.

Theorem 2. One has $S_{f}(P)=T_{f}(P)+O_{\varepsilon, f}\left(P^{5 / 3+\varepsilon}\right)$.

Note that $T_{f}(P)$ counts the number of integral points with $\mathbf{x} \in[-P, P]^{4}$ lying on the union of the three affine planes defined in (1.2), whence $T_{f}(P)=$ $(8+\delta) P^{2}+O(P)$, where $\delta=4$ when $3 a \mid 2 b$, and $\delta$ is zero otherwise.

Next, when $g(s, t)=a s^{3}+b s^{2} t+c s t^{2}+d t^{3}$ is a non-degenerate cubic form with integral coefficients, and $P$ is a positive real number, denote by $N_{g}(P)$ the number of integral solutions of the equation

$$
g\left(x_{1}, x_{0}\right)+g\left(x_{2}, x_{0}\right)=g\left(x_{3}, x_{0}\right)+g\left(x_{4}, x_{0}\right),
$$

with $\left|x_{i}\right| \leqslant P(0 \leqslant i \leqslant 4)$. Also, let $M_{g}(P)$ denote the number of integral 5-tuples $\mathbf{x}$ satisfying one of the conditions

$$
\left\{x_{1}, x_{2}\right\}=\left\{x_{3}, x_{4}\right\} \quad \text { and } 3 a\left(x_{1}+x_{2}\right)+2 b x_{0}=3 a\left(x_{3}+x_{4}\right)+2 b x_{0}=0,
$$

with $\left|x_{i}\right| \leqslant P(0 \leqslant i \leqslant 4)$. In Sect. 4 we establish the following estimate for the number of integral points on the cubic hypersurface determined by (1.3).

Theorem 3. When $a \neq 0$, one has $N_{g}(P)=M_{g}(P)+O_{\varepsilon, g}\left(P^{7 / 3+\varepsilon}\right)$.

Notice that $M_{g}(P)$ essentially counts the number of rational points lying on the projective planes embedded in the hypersurface (1.3), whence $M_{g}(P)=$ $\kappa P^{3}+O\left(P^{2}\right)$ for a suitable real number $\kappa$ satisfying $\kappa \geqslant 16$. The precise value of $\kappa$ is a little more complicated to determine than the corresponding constant $8+\delta$ above, and we choose not to calculate this value explicitly within this paper.

We finish by noting that the mean value estimate provided by Theorem 2 has applications to the problem of providing estimates for the number of values of a 
given cubic polynomial which are not represented in the expected number of ways as the sum of seven cubes of natural numbers. Such matters are discussed in Sect. 8 of BRÜDERn, KAWADA and WOOLEY [1]. We avoid discussing details here, leaving it to the reader to employ Theorem 2 within the latter methods so as to obtain the desired conclusion.

Throughout this paper, implicit constants occurring in Vinogradov's notation $\ll$ and $\gg$, and in Landau's notation, will depend at most on the small positive number $\varepsilon$, and quantities occurring as subscripts to the latter notations, unless indicated otherwise. Also, we use vector notation for brevity. Thus, for example, the triple $\left(d_{1}, d_{2}, d_{3}\right)$ will be abbreviated simply to $\mathbf{d}$. In an effort to simplify our exposition, we adopt the convention that whenever $\varepsilon$ appears in a statement, we are implicitly asserting that the statement holds for each $\varepsilon>0$. Note that the "value" of $\varepsilon$ may consequently change from statement to statement.

\section{The Auxiliary Equation}

The object of this section is to provide an estimate for the number of solutions of a certain auxiliary diophantine equation, this estimate being fundamental to our proofs of Theorems 1 and 2, and motivating also our proof of Theorem 3. Our proof of the aforementioned estimate borrows heavily from the treatment of Wooley [6], but is complicated by the presence of more general cubic polynomials, and also the possibility that the underlying variables may be either positive or negative. Before proceeding further, we require some notation.

When $a$ is an integer, denote by $t(x ; a)$ the polynomial $x^{3}+a x$. Let $X$ be a real number sufficiently large in terms of $a$, and denote by $U_{a}(X)$ the number of integral solutions of the equation

$$
t\left(x_{1} ; a\right)+t\left(x_{2} ; a\right)=t\left(x_{3} ; a\right)+t\left(x_{4} ; a\right),
$$

with

$$
\begin{gathered}
\left|t\left(x_{1} ; a\right)+t\left(x_{2} ; a\right)\right|=\left|t\left(x_{3} ; a\right)+t\left(x_{4} ; a\right)\right| \leqslant X, \\
\left\{x_{1}, x_{2}\right\} \neq\left\{x_{3}, x_{4}\right\}, \quad x_{1}+x_{2} \neq 0, \quad x_{3}+x_{4} \neq 0 .
\end{gathered}
$$

Lemma 2.1. One has $U_{a}(X) \ll X^{5 / 9+\varepsilon}$.

Proof. Let $T_{a}\left(P, Q_{1}, Q_{2} ; h\right)$ denote the number of integral solutions of the simultaneous equations

$$
\begin{aligned}
t\left(x_{1} ; a\right)+t\left(x_{2} ; a\right) & =t\left(x_{3} ; a\right)+t\left(x_{4} ; a\right) \\
x_{1}+x_{2} & =x_{3}+x_{4}+h
\end{aligned}
$$

with $x_{i} \notin\left\{x_{3}, x_{4}\right\}(i=1,2)$,

$$
\begin{gathered}
Q_{1} \leqslant\left|x_{1}\right| \leqslant\left|x_{2}\right| \leqslant 2 Q_{1}, \quad Q_{2} \leqslant\left|x_{3}\right| \leqslant\left|x_{4}\right| \leqslant 2 Q_{2}, \\
1 \leqslant\left|x_{1}+x_{2}\right| \leqslant \min \left\{P^{3} Q_{1}^{-2}, 4 Q_{1}\right\}, \quad 1 \leqslant\left|x_{3}+x_{4}\right| \leqslant \min \left\{P^{3} Q_{2}^{-2}, 4 Q_{2}\right\} .
\end{gathered}
$$

Observe on the one hand that when $h=0$, it follows from (2.4) that

$$
x_{1}^{i}+x_{2}^{i}=x_{3}^{i}+x_{4}^{i} \quad(i=1,3),
$$


whence $x_{1}+x_{2}=x_{3}+x_{4}=0$ or $\left\{x_{1}, x_{2}\right\}=\left\{x_{3}, x_{4}\right\}$. Suppose, on the other hand, that $h \neq 0$ and yet $x_{1}=x_{3}$ or $x_{1}=x_{4}$, or else $x_{2}=x_{3}$ or $x_{2}=x_{4}$. By relabelling variables, there is no loss of generality in supposing that $x_{2}=x_{4}$, and then it follows from (2.4) that one has

$$
x_{1}^{3}+a x_{1}=x_{3}^{3}+a x_{3} \text { and } x_{1}=x_{3}+h .
$$

Thus, since the hypothesis $h \neq 0$ implies that $x_{1} \neq x_{3}$, we deduce that

$$
x_{1}^{2}+x_{1} x_{3}+x_{3}^{2}+a=0 .
$$

The number of integral solutions of the latter equation is $O\left(1+|a|^{\varepsilon}\right.$ ) (see, for example, Estermann [2]), and given any fixed choice for $x_{1}$ and $x_{3}$, one finds from (2.7) that $h$ is uniquely determined. Thus we conclude that the total number of solutions $\mathbf{x}, h$ of the system (2.4) satisfying (2.5) and (2.6), with $h \neq 0$ and $x_{i} \in\left\{x_{3}, x_{4}\right\}$ for $i=1$ or 2 , is $O\left(\min \left\{Q_{1}, Q_{2}\right\}\right)$.

Next we observe that since

$$
4(t(x ; a)+t(y ; a))=(x+y)\left(3(x-y)^{2}+(x+y)^{2}+4 a\right),
$$

it follows that whenever

$$
|t(x ; a)+t(y ; a)| \leqslant X
$$

and $X$ is large, then necessarily

$$
|x+y| \max \left\{|x|^{2},|y|^{2}\right\} \leqslant 4 X .
$$

Suppose then that $\mathbf{x}$ is any solution of (2.1) satisfying (2.2) and (2.3). By relabelling variables we may suppose that $\left|x_{1}\right| \leqslant\left|x_{2}\right|$ and $\left|x_{3}\right| \leqslant\left|x_{4}\right|$. Thus, if $\mathbf{x}$ satisfies the additional condition (2.5), then we have

$$
1 \leqslant\left|x_{1}+x_{2}\right| \leqslant \min \left\{4 X Q_{1}^{-2}, 4 Q_{1}\right\} \quad \text { and } \quad 1 \leqslant\left|x_{3}+x_{4}\right| \leqslant \min \left\{4 X Q_{2}^{-2}, 4 Q_{2}\right\} .
$$

Interchanging the roles of $\left\{x_{1}, x_{2}\right\}$ and $\left\{x_{3}, x_{4}\right\}$ if necessary, we therefore deduce that when $X$ is large, one has

$$
U_{a}(X) \ll(\log X)^{3} \max _{\substack{1 \leqslant Q_{2} \leqslant Q_{1} \leqslant 2^{1 / 2} \\ 1 \leqslant H \leqslant 8 X^{1 / 3}}}\left(Q_{1}+\sum_{H \leqslant|h| \leqslant 2 H} T_{a}\left(2 X^{1 / 3}, Q_{1}, Q_{2} ; h\right)\right) .
$$

Suppose next that $Q_{2} \leqslant Q_{1}$, let $h$ be a non-zero integer, and let $\mathbf{x}$ be any solution of the system (2.4) counted by $T_{a}\left(P, Q_{1}, Q_{2} ; h\right)$. Plainly,

$$
\left(x_{1}+x_{2}-x_{3}\right)^{3}-\left(t\left(x_{1} ; a\right)+t\left(x_{2} ; a\right)-t\left(x_{3} ; a\right)\right)=\left(x_{4}+h\right)^{3}-t\left(x_{4} ; a\right) .
$$

On making the substitution

$$
u_{1}=x_{1}+x_{2}, \quad u_{2}=x_{1}-x_{3}, \quad u_{3}=x_{2}-x_{3}, \quad y=2 x_{4}+h,
$$

moreover, one finds that the $x_{i}$ are uniquely determined from the integers $u_{j}, y$ and $h$. It follows that $T_{a}\left(P, Q_{1}, Q_{2} ; h\right)$ is bounded above by $V_{4 a}\left(P, Q_{1}, Q_{2} ; h\right)$, where $V_{b}\left(P, Q_{1}, Q_{2} ; h\right)$ denotes the number of solutions of the system

$$
\begin{aligned}
12 u_{1} u_{2} u_{3} & =h\left(3 y^{2}+h^{2}+b\right) \\
u_{1}+u_{2}+u_{3} & =y+h
\end{aligned}
$$


with

$$
1 \leqslant\left|u_{1}\right| \leqslant P^{3} Q_{1}^{-2}, \quad 1 \leqslant\left|u_{2}\right| \leqslant 4 Q_{1}, \quad 1 \leqslant\left|u_{3}\right| \leqslant 4 Q_{1}, \quad|y| \leqslant 8 Q_{1} .
$$

Let $V_{b}^{(1)}\left(P, Q_{1}, Q_{2} ; h\right)$ denote the number of solutions of the system (2.9) satisfying (2.10) and the condition

$$
h^{2}+3\left(u_{i}-u_{j}\right)^{2}+b=0
$$

for some $i$ and $j$ with $1 \leqslant i<j \leqslant 3$. Also, let $V_{b}^{(2)}\left(P, Q_{1}, Q_{2} ; h\right)$ denote the number of solutions of the system (2.9) satisfying (2.10) and the condition

$$
3 u_{i}^{2}-3 h u_{i}+h^{2}+b=0
$$

for some $i$ with $1 \leqslant i \leqslant 3$. Finally, denote by $V_{b}^{(3)}\left(P, Q_{1}, Q_{2} ; h\right)$ the number of solutions of the system (2.9) satisfying (2.10), and neither (2.11) nor (2.12) for any $i, j$ with $i \neq j$. Then plainly,

$$
V_{b}\left(P, Q_{1}, Q_{2} ; h\right) \leqslant \sum_{i=1}^{3} V_{b}^{(i)}\left(P, Q_{1}, Q_{2} ; h\right) .
$$

We first bound

$$
W_{1}=\sum_{H \leqslant|h| \leqslant 2 H} V_{b}^{(1)}\left(P, Q_{1}, Q_{2} ; h\right) .
$$

Let $\mathbf{u}, y, h$ be a solution of the system (2.9) counted in the latter sum. Then without loss of generality we may suppose that (2.11) holds with $i=1$ and $j=2$. If $b \geqslant 0$, then there are plainly no solutions with $h \neq 0$. If $b<0$, meanwhile, then the number of possible choices for $h$ and $u_{1}-u_{2}$ is $O\left(|b|^{\varepsilon}\right)$, by making use of estimates for the number of representations of an integer by a binary quadratic form (see Estermann [2]). Fix any one such choice, and any one of the $O\left(Q_{1}\right)$ possible choices for $u_{2}$. Then we may eliminate $y$ between the two equations of the system (2.9), and deduce that $u_{3}$ satisfies a non-trivial polynomial equation. Thus there are $O(1)$ possible choices for $u_{3}$, and by back substitution there are also $O(1)$ possible choices for $y$. Thus the total number of solutions in this case is

$$
W_{1} \ll Q_{1}|b|^{\varepsilon} \text {. }
$$

Next we tackle

$$
W_{2}=\sum_{H \leqslant|h| \leqslant 2 H} V_{b}^{(2)}\left(P, Q_{1}, Q_{2} ; h\right) .
$$

Let $\mathbf{u}, y, h$ be a solution of the system (2.9) counted in the latter sum. Then without loss of generality we may suppose that (2.12) holds with $i=1$, and hence that

$$
3\left(2 u_{1}-h\right)^{2}+h^{2}+4 b=0 .
$$

If $b \geqslant 0$, then there are no solutions of the latter equation with $h \neq 0$. If $b<0$, on the other hand, we find that the number of possible choices for $h$ and $2 u_{1}-h$ is $O\left(|b|^{\varepsilon}\right)$ in a similar manner to the case above. Fix any one such choice, and any of 
the $O\left(Q_{1}\right)$ possible choices for $y$. Then the first equation of (2.9) shows that $u_{1}, u_{2}, u_{3}$ are divisors of the fixed integer $h\left(3 y^{2}+h^{2}+b\right)$. The latter integer is nonzero, moreover, because the conditions (2.10) ensure that $12 u_{1} u_{2} u_{3} \neq 0$. Thus a standard estimate for the divisor function shows that the number of possible choices for $\mathbf{u}$ is $O\left(\left(|b| H Q_{1}\right)^{\varepsilon}\right)$, whence

$$
W_{2} \ll Q_{1}^{1+\varepsilon}(|b| H)^{\varepsilon} .
$$

Finally we negotiate the estimation of $V_{b}^{(3)}\left(P, Q_{1}, Q_{2} ; h\right)$. Let $h$ be a non-zero integer, and suppose that $\mathbf{u}, y$ is a solution of the system (2.9) counted by $V_{b}^{(3)}\left(P, Q_{1}, Q_{2} ; h\right)$. Write

$$
\begin{array}{cll}
d_{3}=\left(u_{3}, h\right), & d_{2}=\left(u_{2}, h / d_{3}\right), & d_{1}=\left(u_{1}, h /\left(d_{2} d_{3}\right)\right), \\
g=h /\left(d_{1} d_{2} d_{3}\right), & f=12 / g \quad \text { and } \quad v_{i}=u_{i} / d_{i} \quad(1 \leqslant i \leqslant 3) .
\end{array}
$$

It follows from (2.9) that $f$ and $g$ are integers, and thus we conclude that $u_{i}=v_{i} d_{i}$ $(1 \leqslant i \leqslant 3)$, and

$$
h=g d_{1} d_{2} d_{3}, \quad f g=12 .
$$

On recalling (2.11) and (2.12), moreover, we have also the conditions

$$
\begin{array}{r}
\left(g d_{1} d_{2} d_{3}\right)^{2}+3\left(d_{i} v_{i}-d_{j} v_{j}\right)^{2}+b \neq 0 \quad(1 \leqslant i<j \leqslant 3), \\
3\left(d_{i} v_{i}\right)^{2}-3\left(g d_{1} d_{2} d_{3}\right)\left(d_{i} v_{i}\right)+\left(g d_{1} d_{2} d_{3}\right)^{2}+b \neq 0 \quad(1 \leqslant i \leqslant 3) .
\end{array}
$$

On substituting into (2.9), and eliminating $y$ by employing the linear equation, we deduce that $V_{b}^{(3)}\left(P, Q_{1}, Q_{2} ; h\right)$ is bounded above by $W_{3}(h)$, where $W_{3}(h)$ denotes the number of solutions of the system

$$
f v_{1} v_{2} v_{3}=3\left(d_{1} v_{1}+d_{2} v_{2}+d_{3} v_{3}-g d_{1} d_{2} d_{3}\right)^{2}+\left(g d_{1} d_{2} d_{3}\right)^{2}+b
$$

with $\mathbf{v}, \mathbf{d}, f$ and $g$ satisfying (2.18)-(2.20), and

$$
1 \leqslant\left|v_{1}\right| \leqslant P^{3}\left(d_{1} Q_{1}^{2}\right)^{-1}, \quad 1 \leqslant\left|v_{2}\right| \leqslant 4 Q_{1} d_{2}^{-1}, \quad 1 \leqslant\left|v_{3}\right| \leqslant 4 Q_{1} d_{3}^{-1} .
$$

For a fixed non-zero integer $h$, an elementary divisor function estimate reveals that the number of possible choices for $f, g$, d satisfying (2.18) is $O\left(|h|^{\varepsilon}\right)$. Fix any one such choice, and consider a fixed choice of $v_{1}$ satisfying (2.22) and the inequality (2.20) with $i=1$. Consider now any solution $v_{2}, v_{3}$ of the equation (2.21) satisfying (2.22) and the inequality (2.19) with $i=2$ and $j=3$. The equation (2.21) takes the shape

$$
6 d_{2}^{2} v_{2}^{2}+6 d_{3}^{2} v_{3}^{2}+2 A v_{2} v_{3}+12 B d_{2} v_{2}+12 B d_{3} v_{3}+C=0
$$

where

$$
A=6 d_{2} d_{3}-f v_{1}, \quad B=d_{1} v_{1}-g d_{1} d_{2} d_{3}, \quad C=6 B^{2}+2\left(g d_{1} d_{2} d_{3}\right)^{2}+2 b .
$$

Notice that necessarily one has

$$
d_{1} v_{1}-g d_{1} d_{2} d_{3} \neq 0,
$$

for otherwise it follows from (2.21) that

$$
\left(g d_{1} d_{2} d_{3}\right)^{2}+3\left(d_{2} v_{2}-d_{3} v_{3}\right)^{2}+b=0,
$$


contradicting (2.19) when $i=2$ and $j=3$. Next, on completing the square we obtain from (2.23) the equation

$$
\Phi^{2}+D \Psi^{2}=n
$$

where

$$
\begin{gathered}
D=36 d_{2}^{2} d_{3}^{2}-A^{2}, \quad n=\left(36 d_{2}^{2} d_{3} B-6 d_{2} A B\right)^{2}-D\left(6 d_{2}^{2} C-36 d_{2}^{2} B^{2}\right), \\
\Phi=D v_{3}+36 d_{2}^{2} d_{3} B-6 d_{2} A B, \quad \Psi=6 d_{2}^{2} v_{2}+A v_{3}+6 d_{2} B .
\end{gathered}
$$

A modest calculation reveals that

$$
D=-f^{2} v_{1}\left(v_{1}-g d_{2} d_{3}\right),
$$

and so it follows from (2.24) that $D \neq 0$. Thus the map $\left(v_{2}, v_{3}\right) \rightarrow(\Phi, \Psi)$ is oneto-one. It follows that the number of possible choices of $v_{2}$ and $v_{3}$ satisfying the above system is bounded above by the number of integral solutions of the equation (2.25) with $|\Phi| \leqslant(100 X)^{3}$ and $|\Psi| \leqslant(100 X)^{3}$. But $D \neq 0$, and a further modest calculation shows that

$$
n=3\left(f d_{2}\right)^{2} v_{1}\left(v_{1}-g d_{2} d_{3}\right)\left(3\left(2 d_{1} v_{1}-g d_{1} d_{2} d_{3}\right)^{2}+\left(g d_{1} d_{2} d_{3}\right)^{2}+4 b\right),
$$

whence by (2.24) and (2.20) with $i=1$, one has $n \neq 0$. Then it follows from the elementary theory of binary quadratic forms (see Estermann [2]) that the number of permissible choices for $\Phi$ and $\Psi$ is $O\left(P^{\varepsilon}\right)$, whence the total number of permissible choices for $v_{1}, v_{2}, v_{3}$ is

$$
\ll \sum_{1 \leqslant\left|v_{1}\right| \leqslant P^{3}\left(d_{1} Q_{1}^{2}\right)^{-1}} P^{\varepsilon} \ll P^{3+\varepsilon}\left(d_{1} Q_{1}^{2}\right)^{-1} .
$$

By interchanging the roles of $\left(v_{1}, v_{2}, v_{3}\right)$ with $\left(v_{2}, v_{3}, v_{1}\right)$, and with $\left(v_{3}, v_{1}, v_{2}\right)$ respectively, one deduces in a similar fashion that the number of permissible choices for $v_{1}, v_{2}, v_{3}$ is

$$
\ll \sum_{1 \leqslant\left|v_{2}\right| \leqslant 4 Q_{1} d_{2}^{-1}} P^{\varepsilon} \ll P^{\varepsilon} Q_{1} d_{2}^{-1},
$$

and also is

$$
\ll \sum_{1 \leqslant\left|v_{3}\right| \leqslant 4 Q_{1} d_{3}^{-1}} P^{\varepsilon} \ll P^{\varepsilon} Q_{1} d_{3}^{-1} .
$$

By combining the latter three bounds, we may conclude that the number of possible choices for $v_{1}, v_{2}, v_{3}$ is

$$
\begin{aligned}
& \ll\left(\left(P^{3+\varepsilon}\left(d_{1} Q_{1}^{2}\right)^{-1}\right)\left(P^{\varepsilon} Q_{1} d_{2}^{-1}\right)\left(P^{\varepsilon} Q_{1} d_{3}^{-1}\right)\right)^{1 / 3} \\
& \ll P^{1+\varepsilon}\left(d_{1} d_{2} d_{3}\right)^{-1 / 3} \ll P^{1+\varepsilon}|h|^{-1 / 3} .
\end{aligned}
$$

Collecting our conclusions thus far together, we have

$$
W_{3}(h) \ll P^{1+\varepsilon}|h|^{\varepsilon-1 / 3},
$$


whence by (2.13)-(2.17),

$$
\begin{aligned}
\sum_{H \leqslant|h| \leqslant 2 H} V_{b}\left(P, Q_{1}, Q_{2} ; h\right) & \ll Q_{1}^{1+\varepsilon}(|b| H)^{\varepsilon}+\sum_{H \leqslant|h| \leqslant 2 H} P^{1+\varepsilon}|h|^{\varepsilon-1 / 3} \\
& \ll P^{1+\varepsilon} H^{2 / 3+\varepsilon}+Q_{1}^{1+\varepsilon} H^{\varepsilon} .
\end{aligned}
$$

Finally, from (2.8) we deduce that

$$
U_{a}(X) \ll X^{\varepsilon}\left(X^{1 / 2}+X^{5 / 9}\right) \ll X^{5 / 9+\varepsilon},
$$

and this completes the proof of the lemma.

\section{The Proof of Theorems 1 and 2}

A linear change of variables reveals that Theorems 1 and 2 are essentially immediate consequences of Lemma 2.1, as we presently demonstrate. We begin with the proof of Theorem 2, which is more transparent than that of Theorem 1.

The proof of Theorem 2. Let $f(s)=a s^{3}+b s^{2}+c s+d$ be a cubic polynomial with integral coefficients. Then plainly $a \neq 0$, and

$$
27 a^{2} f(s)=(3 a s+b)^{3}+A(3 a s+b)+B,
$$

where

$$
A=9 a c-3 b^{2} \quad \text { and } \quad B=27 a^{2} d-9 a b c+2 b^{3} .
$$

It follows that the equation (1.1) holds if and only if

$$
t\left(y_{1} ; A\right)+t\left(y_{2} ; A\right)=t\left(y_{3} ; A\right)+t\left(y_{4} ; A\right),
$$

where we write $y_{i}=3 a x_{i}+b(1 \leqslant i \leqslant 4)$. Since the conditions (1.2) are equivalent, under the above change of variables, to the new conditions

$$
\left\{y_{1}, y_{2}\right\}=\left\{y_{3}, y_{4}\right\} \text { and } y_{1}+y_{2}=y_{3}+y_{4}=0 \text {, }
$$

we deduce from (2.1)-(2.3) that

$$
S_{f}(P)-T_{f}(P) \leqslant U_{A}(X),
$$

where $X$ is a positive real number satisfying $X \ll P^{3}$ (and here the implicit constant depends at most on $a, b, c)$. Thus we deduce from Lemma 2.1 that

$$
S_{f}(P)-T_{f}(P) \ll P^{5 / 3+\varepsilon},
$$

and this completes the proof of Theorem 2 .

The proof of Theorem 1. We make use of the same notation as that applied in the proof of Theorem 2 , and begin by observing that $\nu_{f}^{+}(x)$ is equal to the number of integers $n$ with $|n| \leqslant x$ for which the diophantine equation

$$
n=f\left(x_{1}\right)+f\left(x_{2}\right)=f\left(x_{3}\right)+f\left(x_{4}\right)
$$

is soluble with $\left\{x_{1}, x_{2}\right\} \neq\left\{x_{3}, x_{4}\right\}$. Also, on noting that when

$$
3 a\left(x_{1}+x_{2}\right)+2 b=0
$$


then it follows from (3.1) that

$$
27 a^{2}\left(f\left(x_{1}\right)+f\left(x_{2}\right)\right)=2 B,
$$

we find that there is at most one integer $n$ satisfying (3.5) and (3.6) which contributes to $\nu_{f}^{+}(x)$. Following the change of variables leading to (3.3), therefore, and recalling (2.1)-(2.3), we obtain

$$
\nu_{f}^{+}(x) \leqslant 1+U_{A}\left(27 a^{2} x\right),
$$

whence we conclude from Lemma 2.1 that $\nu_{f}^{+}(x) \ll x^{5 / 9+\varepsilon}$. This completes the proof of Theorem 1 in the first case.

In the second case, we observe that $\nu_{f}^{-}(x)$ is equal to the number of integers $n$ with $|n| \leqslant x$ for which the diophantine equation

$$
n=f\left(x_{1}\right)-f\left(x_{2}\right)=f\left(x_{3}\right)-f\left(x_{4}\right)
$$

is soluble with

$$
\left(x_{1}, x_{2}\right) \neq\left(x_{3}, x_{4}\right) \text { and }\left(x_{3}, x_{4}\right) \neq\left(-x_{2}-2 b /(3 a),-x_{1}-2 b /(3 a)\right) .
$$

On noting that when $x_{1}=x_{2}$ one necessarily has $f\left(x_{1}\right)-f\left(x_{2}\right)=0$, we find that there is precisely one integer, namely 0 , satisfying (3.7) and $x_{1}=x_{2}$ which contributes to $\nu_{f}^{-}(x)$. Following the change of variables leading to (3.3), therefore, and replacing $y_{i}$ by $-y_{i}$ therein for $i=2,4$, it follows from (2.1)-(2.3) that

$$
\nu_{f}^{-}(x) \leqslant 1+U_{A}\left(27 a^{2} x\right) .
$$

The desired conclusion $\nu_{f}^{-}(x) \ll x^{5 / 9+\varepsilon}$ again follows from Lemma 2.1 , and this completes the proof of Theorem 1 in the second case.

\section{A Homogeneous Equation}

We next attend to the proof of Theorem 3. Let $g(s, t)=a s^{3}+b s^{2} t+c s t^{2}+d t^{3}$ be a non-degenerate cubic form with integral coefficients, and with $a \neq 0$. Observe first that

$$
27 a^{2} g(s, t)=(3 a s+b t)^{3}+A(3 a s+b t) t^{2}+B t^{3},
$$

where $A$ and $B$ are given by (3.2). Since $a \neq 0$, the mapping $\mathbf{x} \rightarrow \mathbf{y}$ given by $y_{i}=3 a x_{i}+b x_{0}(1 \leqslant i \leqslant 4)$ and $y_{0}=x_{0}$, is one-to-one. Also, the conditions (1.4) are equivalent under the above change of variables to the the new conditions (3.4). Denote by $\tau_{\alpha}(x ; z)$ the polynomial $x^{3}+\alpha x z^{2}$. Then on recalling the notation of Sect. 1, we may conclude thus far that

$$
N_{g}(P)-M_{g}(P) \leqslant U_{A}^{*}((3|a|+|b|) P),
$$

where $U_{A}^{*}(X)$ denotes the number of integral solutions of the equation

$$
\tau_{A}\left(y_{1} ; y_{0}\right)+\tau_{A}\left(y_{2} ; y_{0}\right)=\tau_{A}\left(y_{3} ; y_{0}\right)+\tau_{A}\left(y_{4} ; y_{0}\right)
$$

with $\left|y_{i}\right| \leqslant X(0 \leqslant i \leqslant 4)$, and with $\mathbf{y}$ satisfying none of the conditions (3.4).

When $A=0$ the equation (4.2) becomes $y_{1}^{3}+y_{2}^{3}=y_{3}^{3}+y_{4}^{3}$. Work of HeAtHBROwN [3] shows that the number of integral solutions $y_{1}, y_{2}, y_{3}, y_{4}$ of the latter 
equation with $\left|y_{i}\right| \leqslant X(1 \leqslant i \leqslant 4)$, and with none of the conditions (3.4) holding, is $O\left(X^{4 / 3+\varepsilon}\right)$. On summing over the permissible choices for $y_{0}$, we therefore find that

$$
U_{0}^{*}(X) \ll X^{7 / 3+\varepsilon} \text {. }
$$

We may consequently suppose henceforth that $A$ is non-zero.

Observe that for each fixed choice of $y_{0}$, the equation (4.2) takes the shape (2.1). We may therefore imitate the proof of Lemma 2.1, regarding $y_{0}$ as fixed and ultimately summing over the available choices for $y_{0}$. On noting that each variable lies in $[-X, X]$ instead of the more liberal range implied by (2.2), we arrive at the estimate

$$
U_{A}^{*}(X) \ll X^{2+\varepsilon}+(\log X) \max _{1 \leqslant H \leqslant 2 X} \sum_{H \leqslant|h| \leqslant 2 H} W(h),
$$

where $W(h)$ denotes the number of solutions of the system

$$
f v_{1} v_{2} v_{3}=3\left(d_{1} v_{1}+d_{2} v_{2}+d_{3} v_{3}-g d_{1} d_{2} d_{3}\right)^{2}+\left(g d_{1} d_{2} d_{3}\right)^{2}+4 A w^{2},
$$

with $w, \mathbf{v}, \mathbf{d}, f$ and $g$ satisfying (2.18) and

$$
|w| \leqslant X, \quad 1 \leqslant\left|v_{i}\right| \leqslant 2 X / d_{i} \quad(1 \leqslant i \leqslant 3) .
$$

For a fixed non-zero integer $h$, an elementary divisor function estimate shows that the number of possible choices for $f, g, \mathbf{d}$ satisfying (2.18) is $O\left(|h|^{\varepsilon}\right)$. Fix any one such choice, and let $W_{1}(f, g, \mathbf{d})$ denote the number of solutions $\mathbf{v}, w$ of (4.5) with (4.6), and satisfying the additional equation

$$
\left(g d_{1} d_{2} d_{3}\right)^{4}=12\left(d_{i} v_{i}\right)\left(d_{j} v_{j}\right)\left(d_{i} v_{i}-g d_{1} d_{2} d_{3}\right)\left(d_{j} v_{j}-g d_{1} d_{2} d_{3}\right),
$$

for some $i$ and $j$ with $1 \leqslant i<j \leqslant 3$. Let $W_{2}(f, g, \mathbf{d})$ denote the corresponding number of solutions in which (4.7) is satisfied for no $i$ and $j$ with $1 \leqslant i<j \leqslant 3$.

Given a solution $\mathbf{v}, w$ counted by $W_{1}(f, g, \mathbf{d})$, we may relabel variables in such a way that, without loss of generality, the equation (4.7) holds with $i=1$ and $j=2$. On noting that the left hand side of (4.7) is a fixed non-zero integer, a standard divisor function estimate reveals that the number of possible choices for $v_{1}$ and $v_{2}$ is $O\left(|h|^{\varepsilon}\right)$. Fixing any one such choice, the variables $v_{3}$ and $w$ satisfy the non-trivial equation (4.5). Thus, given any fixed choice of $w$, we find that there are $O(1)$ possible choices for $v_{3}$, and consequently,

$$
W_{1}(f, g, \mathbf{d}) \ll|h|^{\varepsilon} X .
$$

Consider next a solution $\mathbf{v}, w$ counted by $W_{2}(f, g, \mathbf{d})$. For any fixed choice of $v_{1}, v_{2}$ satisfying (4.6), consider any solution $w, v_{3}$ of the equation (4.5) satisfying (4.6). The equation (4.5) takes the shape

with

$$
\Phi^{2}+3 A \Psi^{2}=n,
$$

$$
\begin{aligned}
n= & \left(f v_{1} v_{2}-6 d_{3}\left(d_{1} v_{1}+d_{2} v_{2}-g d_{1} d_{2} d_{3}\right)\right)^{2}-3\left(2 g d_{1} d_{2} d_{3}^{2}\right)^{2} \\
& \quad-36 d_{3}^{2}\left(d_{1} v_{1}+d_{2} v_{2}-g d_{1} d_{2} d_{3}\right)^{2}, \\
\Phi=6 & d_{3}^{2} v_{3}-f v_{1} v_{2}+6 d_{3}\left(d_{1} v_{1}+d_{2} v_{2}-g d_{1} d_{2} d_{3}\right), \quad \Psi=4 d_{3} w .
\end{aligned}
$$


Since $n$ takes the shape

$$
n=f^{2} v_{1} v_{2}\left(v_{1}-g d_{2} d_{3}\right)\left(v_{2}-g d_{1} d_{3}\right)-12\left(g d_{1} d_{2}\right)^{2} d_{3}^{4},
$$

and (4.7) is not satisfied with $i=1$ and $j=2$ in the present circumstances, one necessarily has that $n$ is non-zero. Plainly, moreover, the map $\left(v_{3}, w\right) \rightarrow(\Phi, \Psi)$ is one-to-one. It follows that the number of possible choices of $v_{3}$ and $w$ satisfying the system (4.5), (4.6) is bounded above by the number of integral solutions of the equation (4.9) with $|\Phi| \leqslant(100 X)^{2}$ and $|\Psi| \leqslant(100 X)^{2}$, whence the elementary theory of binary quadratic forms (see EsTERMANN [2]) shows that the number of permissible choices for $\Phi$ and $\Psi$ is $O\left(X^{\varepsilon}\right)$. Hence the total number of permissible choices for $w, \mathbf{v}$ is

$$
\ll \sum_{1 \leqslant\left|v_{1}\right| \leqslant 2 X / d_{1}} \sum_{1 \leqslant\left|v_{2}\right| \leqslant 2 X / d_{2}} X^{\varepsilon} \ll X^{2+\varepsilon}\left(d_{1} d_{2}\right)^{-1} .
$$

By interchanging the roles of the $v_{i}$, one deduces similarly that the number of permissible choices for $w, \mathbf{v}$ is

$$
\ll X^{2+\varepsilon} \min \left\{\left(d_{2} d_{3}\right)^{-1},\left(d_{3} d_{1}\right)^{-1}\right\} .
$$

By combining the latter estimates, we may conclude that

$$
W_{2}(f, g, \mathbf{d}) \ll|h|^{\varepsilon} X^{2+\varepsilon}\left(\left(d_{1} d_{2}\right)\left(d_{2} d_{3}\right)\left(d_{3} d_{1}\right)\right)^{-1 / 3} \ll X^{2+\varepsilon}|h|^{\varepsilon-2 / 3},
$$

so that on recalling (4.8), we obtain the upper bound

$$
W(h) \ll|h|^{\varepsilon} X+X^{2+\varepsilon}|h|^{\varepsilon-2 / 3} .
$$

On recalling (4.3) and (4.4), therefore, we deduce that

$$
U_{A}^{*}(X) \ll X^{7 / 3+\varepsilon}+X^{2+\varepsilon} \max _{1 \leqslant H \leqslant 2 X} \sum_{H \leqslant|h| \leqslant 2 H}\left(X^{-1}+|h|^{-2 / 3}\right) \ll X^{7 / 3+\varepsilon},
$$

whence by (4.1),

$$
N_{g}(P)-M_{g}(P) \ll P^{7 / 3+\varepsilon} .
$$

This completes the proof of Theorem 3 .

\section{References}

[1] Brüdern J, Kawada K, Wooley TD (2000) Additive representation in thin sequences (submitted 1999)

[2] Estermann T (1931) Einige Sätze über quadratfreie Zahlen. Math Ann 105: 653-662

[3] Heath-Brown DR (1997) The density of rational points on cubic surfaces. Acta Arith 79: 17-30

[4] Hooley C (1963) On the representation of a number as a sum of two cubes. Math Z 82: 259-266

[5] Hooley C (1980) On the numbers that are representable as the sum of two cubes. J Reine Angew Math 314: 146-173

[6] Wooley TD (1995) Sums of two cubes. Internat Math Res Notices 181-185

T. D. Wooley

Department of Mathematics

University of Michigan

East Hall, 525 East University Avenue

Ann Arbor, MI 48109-1109

U.S.A.

e-mail: wooley@math.lsa.umich.edu 